\title{
CircSOD2 induced epigenetic alteration drives hepatocellular carcinoma progression through activating JAK2/STAT3 signaling pathway
}

Zhongwei Zhao ${ }^{\dagger}$, Jingjing Song ${ }^{\dagger}$, Bufu Tang, Shiji Fang, Dengke Zhang, Liyun Zheng, Fazong Wu, Yang Gao, Chunmiao Chen, Xianghua Hu, Qiaoyou Weng, Yang Yang, Jianfei Tu* and Jiansong Ji

\begin{abstract}
Background: Emerging evidence suggests that circular RNAs play critical roles in disease development especially in cancers. Previous genome-wide RNA-seq studies found that a circular RNA derived from SOD2 gene was highly upregulated in hepatocellular carcinoma (HCC), however, the role of circSOD2 in HCC remains largely unknown.

Methods: The expression profiling of circSOD2 and microRNA in HCC patients were assessed by Real-Time Quantitative Reverse Transcription PCR (qRT-PCR). SiRNA or CRISPR-CAS9 were used to silence gene expression. The biological function of circSOD2 in HCC was investigated using in vitro and in vivo studies including, trans-well cell migration, cell apoptosis, cell cycle, CCK8, siRNA interference, western blots, and xenograft mouse model. The underlying molecular mechanism was determined by Chromatin Immunoprecipitation quantitative real time PCR (ChIP-qPCR), bioinformatic analysis, biotin-pull down, RNA immunoprecipitation, 5-mc DNA pulldown and luciferase assays.

Results: In accordance with previous sequencing results, here, we demonstrated that circSOD2 was highly expressed in HCC tumor tissues compared with normal liver tissues. Mechanically, we showed that histone writer EP300 and WDR5 bind to circSOD2 promoter and trigger its promoter H3K27ac and H3K4me3 modification, respectively, which further activates circSOD2 expression. SiRNA mediated circSOD2 suppression impaired liver cancer cell growth, cell migration, prohibited cell cycle progression and in vivo tumor growth. By acting as a sponge, circSOD2 inhibits miR-502-5p expression and rescues miR-502-5p target gene DNMT3a expression. As a DNA methyltransferase, upregulated DNMA3a suppresses SOCS3 expression by increasing SOCS3 promoter DNA methylation. This event further accelerates SOCS3 downstream JAK2/STAT3 signaling pathway activation. In addition, we also found that activated STAT3 regulates circSOD2 expression in a feedback way.

(Continued on next page)
\end{abstract}

\footnotetext{
*Correspondence: jianfei1133@163.com; ji_j_s@sina.com

†Zhongwei Zhao and Jingjing Song contributed equally to this work. Key Laboratory of Imaging Diagnosis and Minimally Invasive Intervention Research, The Fifth Affiliated Hospital of Wenzhou Medical University /Affiliated Lishui Hospital of Zhejiang University/ Clinical College of The Affiliated Central Hospital of Lishui University, Lishui 323000, China
} 
(Continued from previous page)

Conclusion: The novel signaling axis circSOD2/miR-502-5p/DNMT3a/JAK2/STAT3/circSOD2 provides a better understanding of HCC tumorigenesis. The molecular mechanism underlying this signaling axis offers new prevention and treatment of HCC.

Keywords: Hepatocellular carcinoma, Circular RNA, JAK2/STAT3, DNMT3a

\section{Background}

Hepatocellular carcinoma (HCC) accounts for $>80 \%$ of liver cancer cases and is the fourth leading cause of cancer-related death worldwide $[1,2]$. The incidence of $\mathrm{HCC}$ varies between different regions with over $85 \%$ are estimated to occur in low or middle-resource countries, particularly in sub-Saharan Africa and Eastern Asia [3, 4]. Despite great advances have been made in the early diagnosis and treatment, the prognosis of $\mathrm{HCC}$ patients is still low due to late diagnosis, recurrence and late stage metastasis [5]. Dysregulation of signaling pathways including IL-6/STAT3, Wnt/ $\beta$-catenin, and PI3K/AKT etc. [6-8] have been reported in HCC. However, the underlying molecular mechanism remains largely unknown. Identifying new molecular target or mechanism that drive HCC development will help us understand its pathogenesis and provide new therapeutic methods.

Recently, great attention has been paid to study the roles of non-coding RNAs in diseases, especially in cancer research [9-13]. Non-coding RNAs are groups of RNAs with little or no protein coding potency, including micro RNA (miRNA), long non-coding RNA (lncRNA), and recently emerged circular RNA (circRNA), etc. Circular RNA is defined by a continuous closed loop structure generated from its precursor mRNA through back splicing [14]. Although most circular RNAs do not encode protein, they can regulate gene expression in many ways. For example, by acting as a sponge, they can regulate miRNA expression $[15,16]$, through inhibiting RNAPII extension, they can regulate gene transcription [17]. In addition, circular RNAs were also reported to participate in a variety of other activities including gene alternative splicing process, histone modification, RNA maturation and protein synthesis, etc. [18-21]. Emerging evidence suggest that abnormal expression of circular RNAs have been implicated in the progression of diverse cancers including HCC [22-25].

Previous genome-wide RNA-seq studies on HCC tumor tissues and its adjacent normal liver tissues revealed that a circular RNA derived from SOD2 gene was highly expressed in tumor tissues compared to its adjacent normal liver tissues [26]. However, the role of circSOD2 in HCC remains unknown. Here, we found that highly expressed circSOD2 promotes liver cancer cell proliferation and is linked to cancer progression in vivo. Mechanically, we demonstrated that, upregulated
circSOD2 suppressed miR-502-5p expression, which further promotes DNMT3a expression and activates JAK2/ STAT3 signaling pathway. In addition, we also showed that STAT3 regulates circSOD2 expression in a feedback way. This study provided a new mechanism through which circSOD2 promotes HCC pathogenesis, and implicated new diagnostic and therapeutic targets.

\section{Material and methods}

Cell lines and patient sample

Cell lines used in this study including normal liver cell line HL-7702, liver cancer cell line HEPG2, HUH7, SKHEP1 and HEP3B were purchased from Chinese Academy of Sciences, Shanghai, China. HEPG2 and SK-HEP1 were grown in MEM supplied with 10\% Fetal Bovine Serum (FBS), HL-7702 and HUH7 were cultured in DMEM supplied with 10\% FBS, HEP3B was grown in RPMI-1640 supplied with 15\% FBS. All cells were maintained at $37^{\circ} \mathrm{C}$ in a humidified incubator with $5 \% \mathrm{CO} 2$. Primary tumor tissues and their paired adjacent normal tissues were obtained from patients who underwent surgery at Fifth Affiliated Hospital of Wenzhou Medical University. Samples were collected, snap freeze with liquid nitrogen and used for further studies. All patients gave written informed consent.

\section{Real-time quantitative reverse transcription PCR (qRT- PCR)}

Cells or tissues were collected, total RNA was extract with Trizol reagent (Thermo Fisher, cat: 15596026). cDNA was generated with iScript ${ }^{\mathrm{Tm}}$ Reverse Transcription Reagents (Bio-rad, cat: 1708841) following manufactures' instructions. cDNA was detected with SYBG Super Real PreMix Plus (Tiangen, Cat: FP205). Relative RNA expression was calculated with 2- $\Delta \Delta$ ct. Primers used in this study were listed in Table 1.

\section{Luciferase assay}

SOCS3 promoter was amplified with primers SOCS3p-F: ccGGTACCGGAGGCCGCGCTCGCGGG and SOCS3p-R: aacAGATCT CGCGCAGCACCAAACTGC C. Amplified fragment was inserted into PGL3-Basic vector between KpnI and BglII cutting sites. pcDNA3myc-dnmt3a was purchased from BIofeng, china. $2 \mu \mathrm{g}$ PGL3-SOCS3p, 0.1 $\mu \mathrm{g}$ Renilla vector, and different doses of PCDNA3-myc-dnmt3a were transfected into HEPG2 
Table 1 Primers used in this study

\begin{tabular}{|c|c|}
\hline Name & $5^{\prime}-3^{\prime}$ \\
\hline qRT-circSOD2-F & AAACCACGATCGTTATGCTG \\
\hline qRT-circSOD2-R & CGTTAGGGCTGAGGTTTGTC \\
\hline CTCFsg-F & caccgCGATCCAAATTTGAACGCCG \\
\hline CTCFsg-R & aaacCGGCGTTCAAATTTGGATCGc \\
\hline STAT3sg-F & caccgAGATTGCCCGGATTGTGGCC \\
\hline STAT3sg-R & aaacGGCCACAATCCGGGCAATCTc \\
\hline EP300sg-F & caccgCTTGGCAAGACTTGCCTGAC \\
\hline EP300sg-R & aaacGTCAGGCAAGTCTTGCCAAGc \\
\hline WDR5sg-F & caccgCAGTGCCTGAAGACGCTCAT \\
\hline WDR5sg-R & aaacATGAGCGTCTTCAGGCACTGc \\
\hline DNMT3asg-F & caccgCCGCTCCGCAGCAGAGCTGC \\
\hline DNMT3asg-R & aaacGCAGCTCTGCTGCGGAGCGGC \\
\hline qRT-GAPDH-F & GGAGCGAGATCCCTCCAAAAT \\
\hline qRT-GAPDH-R & GGCTGTTGTCATACTTCTCATGG \\
\hline K27ac/K4me3-ChIP-qPCR-circSOD2p-F & GCCTATGAGCTGAGGGTAGA \\
\hline K27ac/K4me3-ChIP-qPCR-circSOD2p-R & TGCCTCCTGTCCTGGAATA \\
\hline pGL3-SOCS3p-F & ccGGTACCGGAGGCCGCGCTCGCGGG \\
\hline pGL3-SOCS3p-R & aacAGATCTCGCGCAGCACCAAACTGCC \\
\hline K27ac-SOCS3p-F & GCCTATGAGCTGAGGGTAGA \\
\hline Me-SOCS3p-R & TGCCTCCTGTCCTGGAATA \\
\hline EP300-ChIP-qPCR-circSOD2p-F & TGTGAACCAACTGTTCAGGATAA \\
\hline EP300-ChIP-qPCR-circSOD2p-R & ACTGTTGAGAGAGCACTTGATAC \\
\hline WDR5-ChIP-qPCR-circSOD2p-F & ТТСССАССТТСТТСАСТАСТ \\
\hline WDR5-ChIP-qPCR-circSOD2p-R & GGAAAGAATCCTCTGTTGTCCT \\
\hline Me-qPCR-SOCS3p-F & СССАACTTСТСАТTСАСАСТTTC \\
\hline Me-qPCR-SOCS3p-R & CAGGTCGGCCTCCTAGA \\
\hline qRT-U6-F & GCTTCGGCAGCACATATACTAAAAT \\
\hline qRT-U6-R & CGCTTCACGAATTTGCGTGTCAT. \\
\hline
\end{tabular}

and HUH7 cells by Lipofectamine 2000. Cells were collected $48 \mathrm{~h}$ later. Luciferase activity was determined with Dual-Luciferase reporter assay kit (Promega, cat: E1910).

\section{Cell migration assay}

Cell migration was performed in trans-well chambers (24-well plate, $8-\mu \mathrm{m}$ pore size). In brief, cells were collected by trypsin digestion and washed with PBS twice. Load 100ul containing $1 \times 105$ cells onto the upper chamber and incubate the trans-well plate at $37^{\circ} \mathrm{C}$ and $5 \% \mathrm{CO} 2$ for $24 \mathrm{~h}$. The lower compartment was added with $2 \mathrm{~mL}$ DMEM containing 1\% FBS. 24h later the upper chamber was removed, cells migrated to the lower chamber was fixed with $5 \%$ glutaraldehyde for $10 \mathrm{~min}$ at room temperature and stained with $0.1 \%$ Crystal Violet. Cell numbers in five widefield were counted following crystal violet staining.

\section{Cell proliferation assay}

After transfection for $24 \mathrm{~h}$, cells were collected, and approximately 1000 cells were seeded into 96 well plates. Cells growth was detected by CCK8 assay kit in accordance with manufacturer's instructions. Relative vial cell number was evaluated by spectrofluorometer at wavelength OD450.

\section{Cell cycle}

Cell cycle was measured with Propidium iodide (PI) staining. Cells were harvested, washed with PBS and fixed with $90 \%$ ice cold ethanol at $-20^{\circ} \mathrm{C}$ overnight. The next day, cells were allowed to equilibrate to RT by incubating at RT for $5 \mathrm{~min}$. Cells were then washed with PBS at RT twice and stained with $1 \mathrm{ml}$ PI staining solution $(50 \mu \mathrm{g} / \mathrm{ml}, 1 \mathrm{mg} / \mathrm{ml}$ of RNase A, $0.1 \%$ Triton X-100 in PBS) by incubating at RT for 30 mins in the dark. Flow cytometry was applied to detect cell cycle. 


\section{Cell apoptosis}

Cells were collected, washed with PBS and stained with 7-ADD and FITC labeled Annexin-V at ice for 30mins. After staining cell apoptosis was detected by FACS.

\section{CRISPR-CAS9 mediated gene knockout}

EP300, WDR5, STAT3, DNMT3a, SOCS3 were deleted by CRISPR-CAS9. In brief, sgRNAs were designed with online tools (https://portals.broadinstitute.org/gpp/public/analysis-tools/sgrna-design). Annealed sgRNAs were cloned into pLentiGuide-Puro and co-transfected with VSVG, and PsPAX2 into $293 \mathrm{~T}$ cells with Mirus transfection reagent. Fresh medium was replenished $16 \mathrm{~h}$ later. Supernatant containing released lentivirus were collected and filtered through $0.45 \mathrm{uM}$ filter after $48 \mathrm{~h}$. Collected lentivirus were used to infect target cells stably expressing CAS9. 2 days later, fresh medium containing $3 \mu \mathrm{g} / \mathrm{ml}$ Puromycin was added into cells to deplete uninfected cells for another 3 days. The efficiency of gene knockout was evaluated by western blots.

\section{Chromatin Immunoprecipitation quantitative PCR (ChIP- qPCR)}

Tissues were disrupted with dounce homogenizer to obtain single cell suspension. Cells were crosslinked with $1 \%$ formaldehyde and lysed with cell lysis buffer on ice for $20 \mathrm{~min}$. DNA was then sonicated into $200 \sim 500 \mathrm{bp}$ with bioruptor. Protein A/G beads were used for preclear followed by antibody pull down with shaking at $4{ }^{\circ} \mathrm{C}$ overnight. IgG was used as control. The next day, DNA-protein-antibody complexes were pulled down by Protein A/G beads. Beads were washed, DNA-Protein complexes were reverse crosslinked with Proteinase K, DNA was then purified with DNA purification kit. Quantification PCR was used to determine the enrichment of DNA.

\section{DNA methylation pull down (5-mc DNA pull down)}

The methylation status on SOCS3 promoter was determined with Methylated-DNA IP Kit (Zymo research, Cat: D5101) according to manufacturer's instructions. The enrichment of desired DNA region was evaluated by qPCR.

\section{RNA immunoprecipitation (RIP)}

Cells were harvested and crosslinked with formaldehyde. Cells were then lysed, and chromatin was sonicated. Ago2 antibody or IgG was used to pull down Ago2-RNA complexes with shaking at $4{ }^{\circ} \mathrm{C}$ overnight. The next day, ProteinA/G beads was added to precipitate antibodyAgo2-RNA complexes. Beads were then washed to wash off unbound materials. RNA was purified with RNA purification kit. The enrichment of RNA was determined by qRT-PCR.

\section{In vivo xenograft mouse model}

The animal experiment was strictly conducted based on the protocol of the institutional ethics board of the Fifth Affiliated Hospital of Wenzhou Medical University. Every effort was made to minimize the pain of mice. 4week old $\mathrm{BALB} / \mathrm{C}$ nude mice were purchased from shanghai animal center. siRNA targeting circSOD2 or scramble control siRNA was transfected into HEPG2 cells. Cells were collected $24 \mathrm{~h}$ later. $1 \times 10^{7}$ cells resuspended in 200ul PBS were then subcutaneously injected into the right flanks of BALB/c nude mice. Tumor growth was monitored and measured. The experiment was stopped 5 weeks later.

\section{Western blots}

Cells were harvested and lysed with Radioimmunoprecipitation assay buffer (RIPA buffer). Supernatants containing protein was obtained by centrifuging cell lysis at Maxi speed for $20 \mathrm{~min}$. Protein was then boiled with 3XSDS loading buffer, separated on 9\% SDS-PAGE gel and transferred to nitrocellulose membrane (NC). Primary and secondary antibody were used to detect protein of interest. Signals were visualized with ECL reagent.

\section{Statistic analysis}

Data were analyzed with SPSS software. Two-side T test was used to determine the differences between untreated and treated groups. Cell growth and tumor formation were analyzed with Two way-ANOVA $* P<0.05,{ }^{* *} P<$ $0.01,{ }^{* * *} P<0.001 . P<0.05$ was considered statistically significant.

\section{Results}

CircSOD2 is highly expressed in HCC tumor tissues and liver cancer cell lines

Genome-wide RNA-seq studies on HCC tumor tissues and their adjacent nontumorous liver tissues revealed that hsa_circ_0004662, derived from SOD2 gene was significantly upregulated in HCC tumor tissues [26]. However, its role in HCC is still unknown. To validate RNAseq results, qRT-PCR with divergent primers complementary to circSOD2 exon 1 and 4 (Fig. 1a) was used to detect circSOD2 expression from patients and cell lines. In line with RNA-seq data, circSOD2 expression was significantly upregulated in 18/19 HCC patient's tumor tissues and liver cancer cell lines compared with normal liver tissues and normal liver cells (Fig. 1b-c). High circSOD2 expression was also associated with poor survival (Fig. 1f) and linked to higher grade tumors (Supplemental Table 1). To further characterize circSOD2, RNA was extracted from HEPG2 and HUH7 cells and treated with or without RNase A before reverse transcription. The effect of RNase A on circSOD2 expression was then 


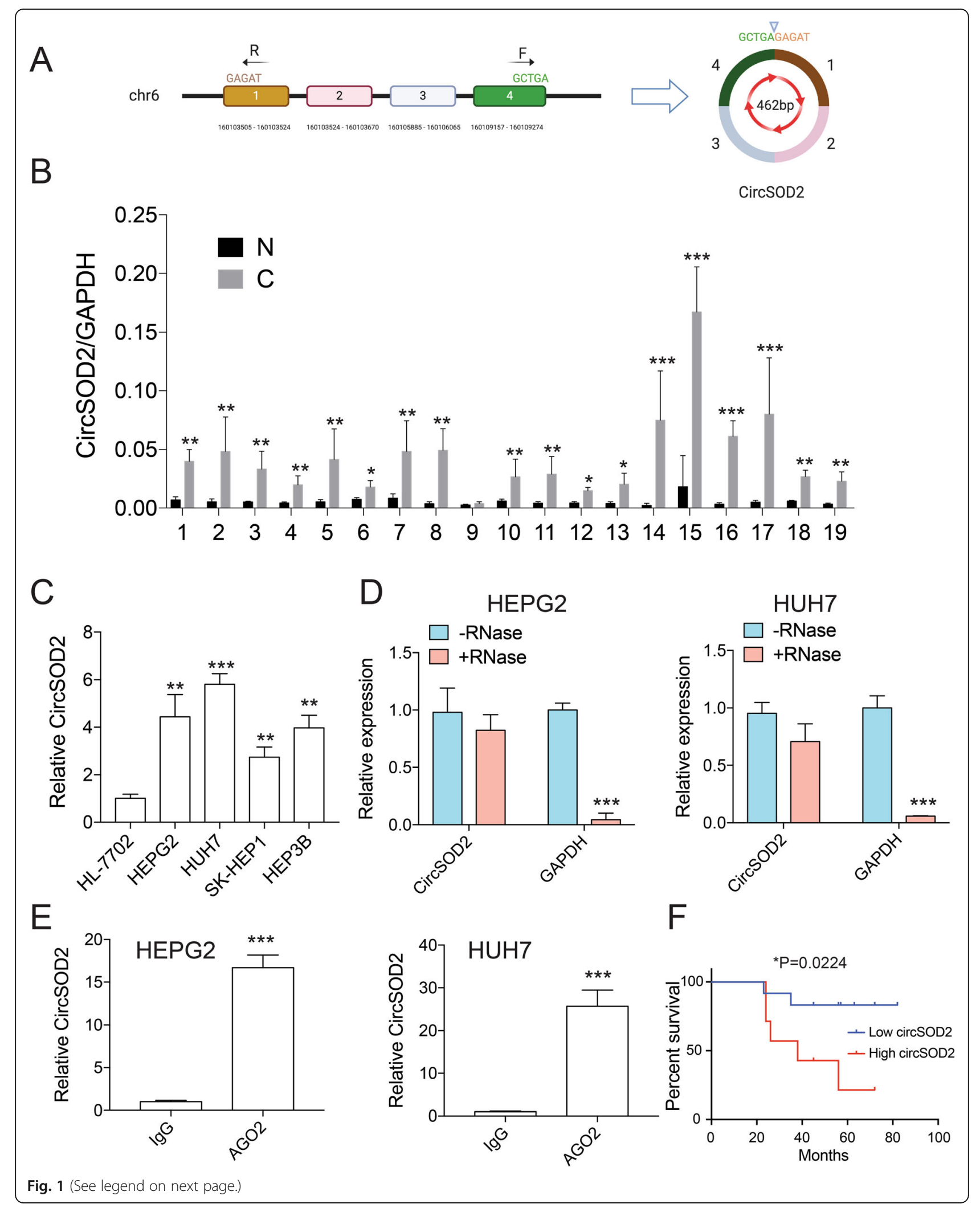


(See figure on previous page.)

Fig. 1 CircSOD2 is highly expressed in HCC tumor tissues and liver cancer cell lines. a Demonstration of circSOD2 formation, F and R are divergent primers used for circSOD2 detection. b RT-qPCR results of circSOD2 expression in 19 HCC patient tumor tissues and adjacent normal liver tissues. N normal, C cancer. c circSOD2 expression in normal liver cell HL-7702 and liver cancer cell line HEPG2, HUH7, SK-HEP1, and HEP3B. d circSOD2 expression after treatment with $1 \mathrm{mg} / \mathrm{ml}$ RNase A, GAPDH was used as control. e circSOD2 expression after pulling down with AGO2 or IgG. $\mathbf{f}$ The overall survival of HCC patients with low or high expression of circSOD2 in HCC tissues were assessed by Kaplan-Meier survival analysis. ${ }^{*} P<0.05,{ }^{*} P<0.01,{ }^{* * *} P<0.001$

examined. Similar to other circRNA, circSOD2 was highly resistant to RNase A digestion. However, the RNA level of GAPDH was greatly impaired after RNase A treatment (Fig. 1d). Data from circular RNA interactome showed that 13 circSOD2 binding sites exist in an RNA binding protein-Ago2 (data not shown), indicating circSOD2 may interact with Ago2 within the cell. To confirm this, RIP assay with Ago2 antibody or IgG control was performed. Indeed, Ago2 significantly precipitated circSOD2 from HEPG2 and HUH7 cells compared to IgG control (Fig. 1e).

\section{CircSOD2 promoter is intensively modified by H3K27ac and $\mathrm{H} 3 \mathrm{~K} 4 \mathrm{me} 3$}

H3K27ac and H3K4me3 modification indicate active gene transcription $[27,28]$. To understand if these modifications contribute to circSOD2 upregulation in HCC. H3K27ac and H3K4me3 ChIP-seq data from HEPG2 cells were then examined. As shown in WashU browser, SOD2 promoter was extensively occupied by H3K27ac and H3K4me3 (Fig. 2a), correlating with high circSOD2 expression. ChIP-qPCR with H3K27ac and H3K4me3 antibodies confirmed higher $\mathrm{H} 3 \mathrm{~K} 27 \mathrm{ac}$ and H3K4me3 modification on circSOD2 promoter from HCC tumor tissues and liver cancer cell lines compared to normal liver tissues (Fig. 2b-c) and normal liver cells (Supplementary figure 1A-B). EP300 and WDR5 mediate H3K27ac and H3K4me3 modification respectively. We next ask if EP300 and WDR5 bind to circSOD2 promoter. ChIP-qPCR was then performed. Similar to H3K27ac and H3K4me3 signal, EP300 and WDR5 were also significantly enriched in circSOD2 promoter from HCC tumor tissues compared to normal liver tissues (Fig. 2d-e). In addition, partial depletion of EP300 or WDR5 (Fig. 2f,i) greatly impaired H3K27ac or H3K4me3 signal on circSOD2 promoter (Fig. 2g,j), circSOD2 expression was also downregulated (Fig. 2h,k). Thus, these results indicate that the enrichment of EP300 and WDR5 on circSOD2 promoter increased its H3K27ac and H3K4me3 modification and circSOD2 expression in HCC.

CircSOD2 promotes in vitro liver cancer cell proliferation and tumorigenesis in vivo

To further characterize the role of circSOD2 in HCC, siRNA targeting the back spliced site of circSOD2 was used to silence circSOD2 expression in liver cancer cells. SiRNA efficiently silenced circSOD2 expression $\sim 6$ fold lower than its original level (Fig. 3a). CircSOD2 downregulation impaired HEPG2 and HUH7 cell growth, and cell migration (Fig. 3b-e). Moreover, cell cycle was also arrested in G0/G1 phase and cell apoptosis was increased following circSOD2 depletion (Fig. 3f-h). The role of circSOD2 in in vivo tumorigenesis was also examined. In accordance with impaired in vitro cell proliferation, silencing circSOD2 also decreased HCC tumor formation in nude mice compared with scramble control (Fig. 3i-j). Taken together, these results suggest that high circSOD2 expression may associate with HCC development.

\section{CircSOD2 suppresses miR-502-5p expression by acting as a sponge}

More and more evidences suggest that, by acting as a sponge, circular RNAs regulate miRNA expression [29, 30]. By searching the circular RNA interactome database, we found that miR-502-5p is a target of circSOD2. The potential interaction site between circSOD2 exon 3 and $5^{\prime}$ miR-502-5p was shown (Fig. 4a). Our previous results showed that Ago2 interacts with circSOD2 in cells. In addition to that, here, we found Ago2 coprecipitated circSOD2 and miR-502-5p from HEPG2 and HUH7 cells (Fig. 4b). To further determine if circSOD2 could directly interact with miR-502-5p. Wild type miR-502-5p or circSOD2-miR-502-5p interaction sites mutated miR-502-5p was labeled with biotin and used to pull down circSOD2 from cell lysates (Fig. 4c-d). As indicated, wild type miR-502-5p efficiently precipitate circSOD2. However, the interaction between mutant miR-502-5p and circSOD2 was almost completely abolished (Fig. 4e). Thus, these results confirmed the direct interaction between circSOD2 and miR-502-5p within liver cancer cells.

To further understand if circSOD2 plays a role in regulating miR-502-5p expression. The expression of miR-502-5p in HCC tumor tissues and liver cancer cells was first investigated. In contrast to circSOD2, miR-502$5 \mathrm{p}$ expression was downregulated in HCC patient tumor tissues and liver cancer cell lines compared with normal liver tissues and normal liver cells (Fig. 4f-g). In addition, the expression level of miR-502-5p was negatively correlated with circSOD2 in HCC tumor tissues (Fig. 4h). 


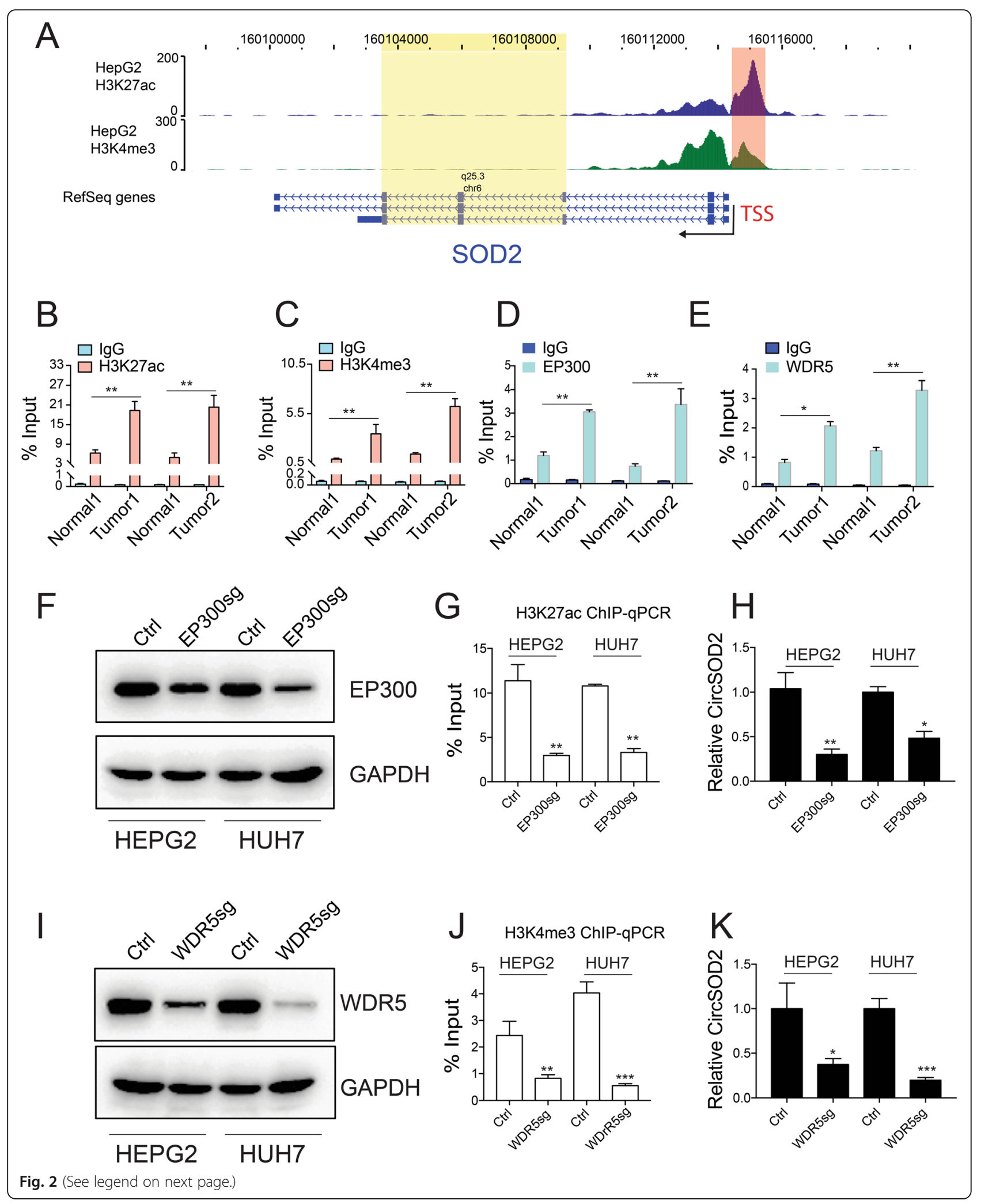


(See figure on previous page.)

Fig. 2 CircSOD2 promoter is intensively modified by H3K27ac and H3K4me3. a H3K27ac and H3K4me3 ChIP-seq results from HEPG2. circSOD2 and SOD2 promoter region were labeled with red, chromosome region spanning circSOD2 transcripts was labeled with yellow. H3K27ac (b) and H3K4me3 (c) ChIP-qPCR signal on circSOD2 promoter and control regions from HCC tumor tissues and adjacent normal liver tissues, lgG was used as control. Enrichment of EP300 (d) and WDR5 (e) on circSOD2 promoter and control regions from HCC tumor tissues and adjacent normal liver tissues. f Western blot results of EP300 after CRIS PR-CAS9 mediated gene depletion, GAPDH was used as control. $\mathbf{g}$ H3K27ac signal on circSOD2 promoter after deleting EP300. $\mathbf{h}$ circSOD2 expression after deleting EP300. i Western blot results of WDR5 after CRISPR-CAS9 mediated gene depletion, GAPDH was used as control. $\mathbf{j}$ H3K4me3 signal on circSOD2 promoter after deleting WDR5. $\mathbf{k}$ circSOD2 expression after deleting WDR5. ${ }^{*} P<0.05,{ }^{* *} P<0.01,{ }^{* * *} P<0.001$

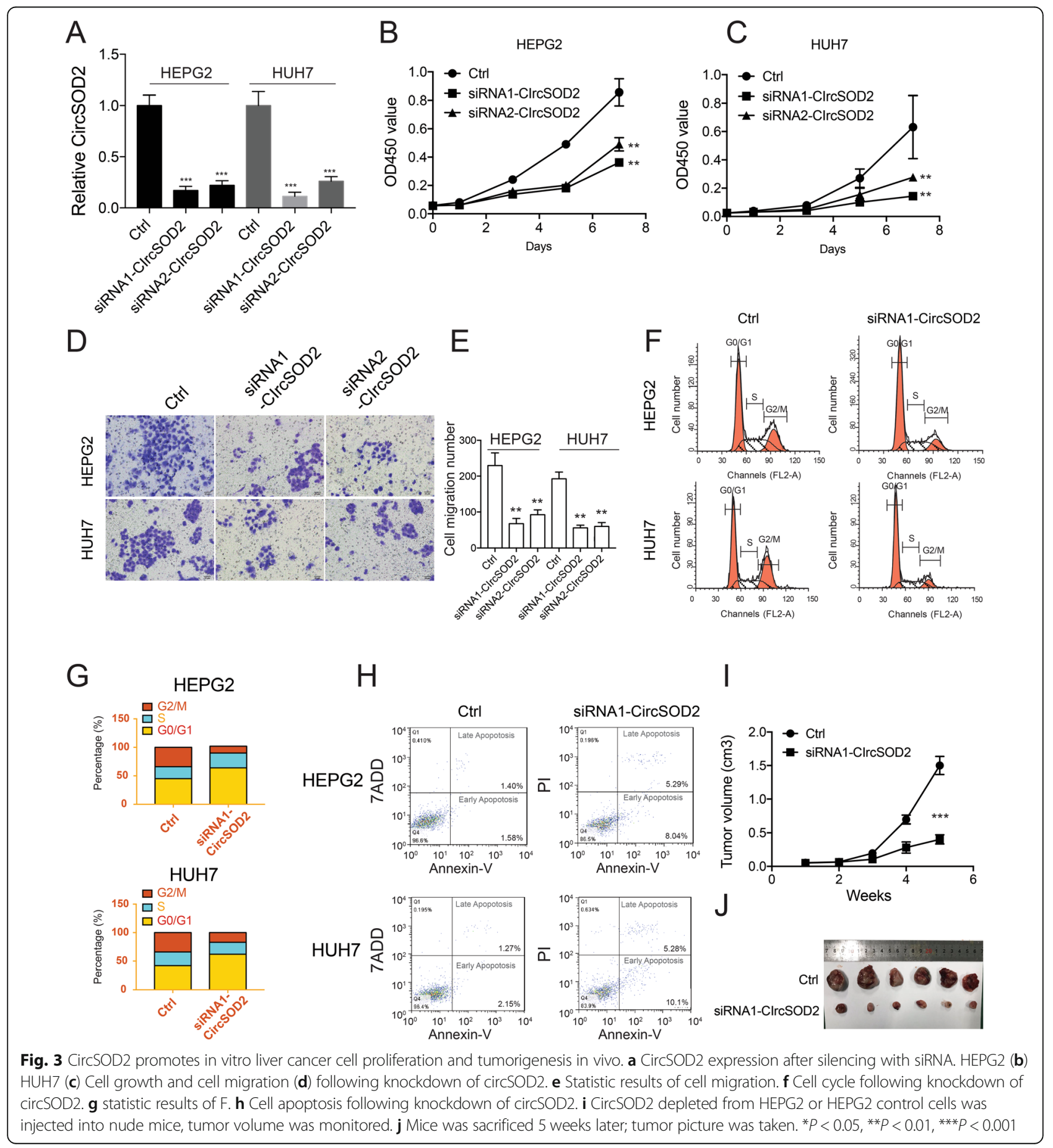




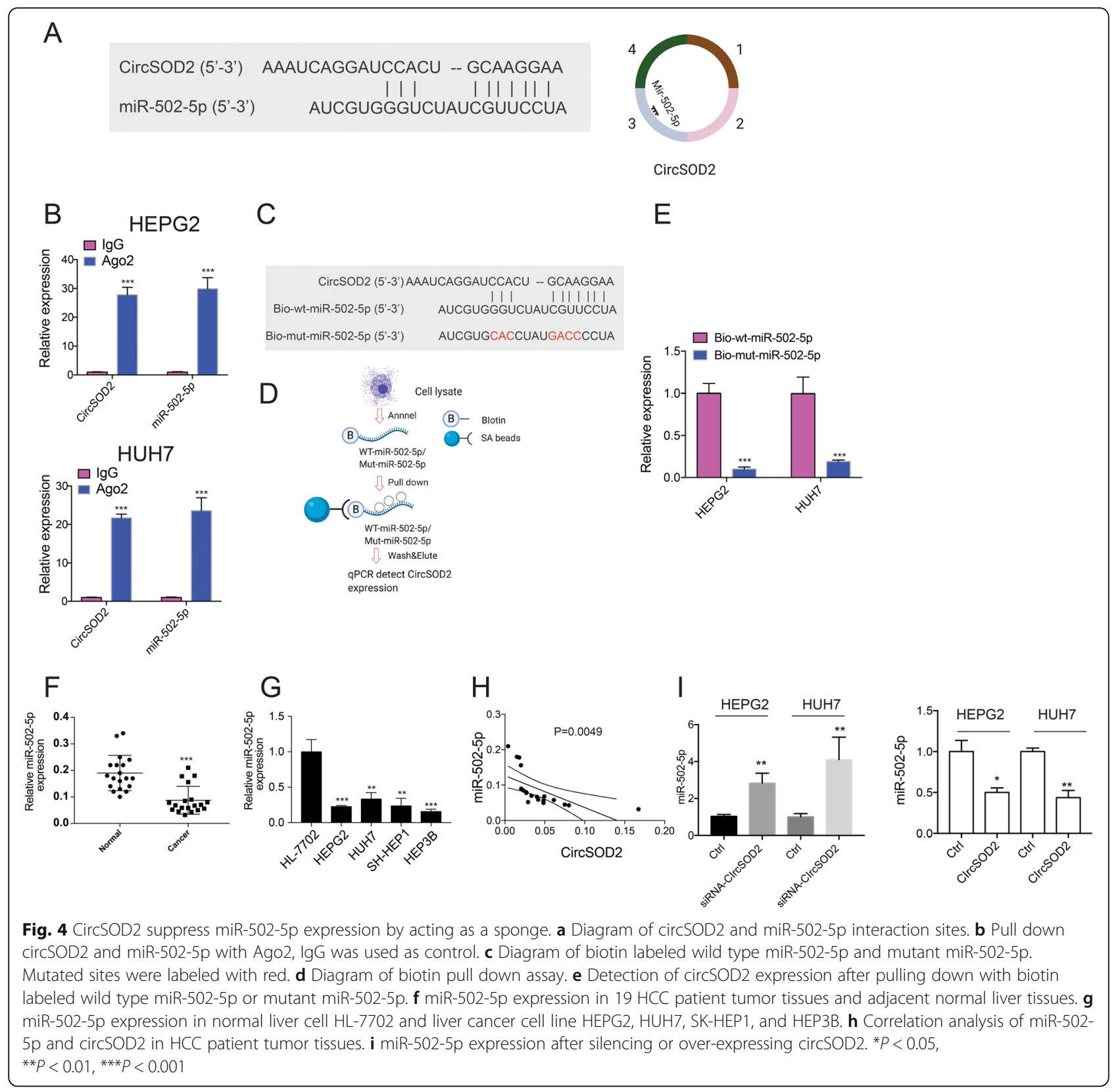

Furthermore, siRNA mediated circSOD2 knockdown greatly upregulated miR-502-5p expression in the meantime, over-expression of circSOD2 suppressed miR-502$5 \mathrm{p}$ expression (Fig. 4i). Above all, these results clearly demonstrate that, by acting as a sponge, circSOD2 suppressed miR-502-5p expression in liver cancer cells.

\section{Overexpression of miR-502-5p downregulates DNMT3a expression}

miRNAs interact with the $3^{\prime}$ untranslated region (3' UTR) of target mRNAs to induce mRNA degradation and translational repression [31]. Database from miRSystem, and TargetScan suggest that DNMT3a, a DNA methyltransferase is a target of miR-502-5p. The interaction site between DNMT3a 3'UTR and miR-502-5p was shown (Fig. 5a). To test if miR-502-5p regulates DNMT3a expression in liver cancer cells, miR-502-5p mimic was introduced into HEPG2 and HUH7 cells (Fig. 5b). Indeed, introducing miR-502-5p mimic into HEPG2 and HUH7 cells significantly downregulated DNMT3a transcription (Fig. 5c), DNMT3a translation was also repressed (Fig. 5d). To confirm the direct interaction between DNMT3a 3'UTR and miR-502-5p. Wild type or mutant DNMT3a 3'UTR was labeled with biotin and used to pull down miR-502-5p from cell lysates. Wild type DNMT3a 3'UTR significantly enriched miR- 
502-5p compared to mutant DNMT3a 3'UTR (Fig. 5e-f). The higher DNMT3a expression in liver cancer cells compared to normal liver cells (Fig. $5 \mathrm{~g}$ ) suggests that downregulation of miR-502-5p in HCC may otherwise facilitate DNMT3a expression.

\section{DNMT3a activates JAK2/STAT3 signaling pathway by suppressing SOCS3 expression}

Dysregulation of IL-6/STAT3 signaling pathway has been implicated in the pathogenesis of HCC [32-34]. To gain insights into the molecular mechanism underlying the role of DNMT3a in HCC, CRISPR-CAS9 was used to delete DNMT3a from HEPG2 and HUH7 cells, the effect of DNMT3a depletion on the expression of genes involved in JAK/STAT3 signaling pathway was examined. DNMT3a was efficiently deleted by CAS9 in HEPG2 and HUH7 cells. Interestingly, JAK2 inhibitor SOCS3 was greatly upregulated, while phosphorylated JAK2, and phosphorylated STAT3 were all downregulated (Fig. 6a), indicating DNMT3a may be involved in SOCS3/pJAK2/pSTAT3 signaling pathway regulation in HCC. Since SOCS3 locates in the upstream of JAK/ STAT signaling pathway. We next asked if DNMT3a could directly regulate SOCS3. Indeed, co-transfecting DNMT3a and SOCS3 promoter driven luciferase vector suppressed luciferase activity in a dose dependent manner (Fig. 6b). However, no effect was observed in SHP1 or SOCS1 promoter activity in the presence of DNMT3a (Supplemental figure 1C-D). DNMT3a is a DNA methyltransferase that modifies CpG methylation and suppresses gene expression. By analyzing SOCS3 promoter, we found that there is an CPG island in the SOCS3 promoter (Fig. 6c), suggesting that DNMT3a may downregulate SOCS3 expression by modifying its promoter methylation status. To test this hypothesis, DNMT3a ChIP-qPCR and DNA methylation pull down assay were performed. DNMT3a was highly enriched in SOCS3 promoter compared to its nearby non-CpG region (Fig. 6d), 5mc antibody also significantly precipitated SOCS3 promoter compared to control (Fig. 6e). Moreover, knocking down DNMT3a decreased SOCS3 promoter methylation in both HEPG2 and HUH7 cells (Fig. 6f). In summary, these results suggest that, in liver cancer cells, DNMT3a upregulation promotes SOCS3 promoter methylation and suppresses SOCS3 expression, which further activates JAK2/STAT3 signaling pathway.

\section{DNMT3a rescues liver cancer cell proliferation impaired by circSOD2 depletion}

To further confirm that DNMT3a is the downstream gene among circSOD2 regulated signaling pathway. CircSOD2 was depleted in the presence or absence of exogenously expressed DNMT3a. In the absence of
DNMT3a, depletion of circSOD2 downregulated DNMT3a and upregulated SOCS3 expression. However, SOCS3 expression was restored when DNMT3a was expressed (Fig. 7a), suggesting that the effect of circSOD2 depletion on upregulating SOCS3 expression was mediated through suppressing DNMT3a. In line with these results, we showed that the expression of circSOD2 was negatively associated with SOCS3 while positively associated with DNMT3a expression in HCC tissues (Supplemental figure 1). In addition to that, we found that hampered liver cancer cell growth and cell migration induced by circSOD2 depletion were also rescued by DNMT3a (Fig. 7b-d). Collectively, these results demonstrate that DNMT3a is the downstream gene of circSOD2 regulated signaling pathway.

\section{STAT3 upregulates circSOD2 expression in a feedback way}

Motif analysis with JASPAR found that eight STAT3 binding sites occupy circSOD2 promoter (Fig. 8a), suggesting that STAT3 may regulate circSOD2 expression. To confirm this, ChIP-qPCR with STAT3 antibody was performed. DNA region that is close to circSOD2 promoter without STAT3 binding sites was used as a control. STAT3 antibody significantly enriched circSOD2 promoter compared to control IgG and control region (Fig. 8b). Depletion of STAT3 also suppressed circSOD2 expression (Fig. 8c-d). These results suggest that STAT3 regulates circSOD2 expression in a feedback way.

\section{Discussion}

Liver cancer poses great threat to human health. Annually, approximately 800,000 new cases are diagnosed with and over 700,000 deaths are linked to liver cancer worldwide [1]. Understanding its underlying pathogenesis mechanism may provide therapeutic targets. In this study, we found that circSOD2, derived from SOD2 was highly expressed in HCC patient tumor tissues and liver cancer cells compared to normal liver tissues and normal liver cells, respectively. Depletion of circSOD2 significantly ceased liver cancer cell growth and impaired in vivo tumorigenesis. Mechanically, we showed that, by acting as a sponge for miR-502-5p, circSOD2 suppressed miR-502-5p expression, which in turn upregulated DNMT3a expression. Elevated DNMT3a suppressed SOCS3 expression and further activates JAK2/STAT3 signaling pathway. In addition, we also found that increased STAT3 in liver cancer cells also regulates circSOD2 expression in a feedback way.

Complexed roles of miR-502 have been revealed in different cancers. For example, hsa-miR-502-5p (miR-502) was reported to inhibit autophagy and tumor growth in colon cancer by suppression of Rab1B [35]. Exogenously introduce miR-502-5p mimic into breast cancer cells 


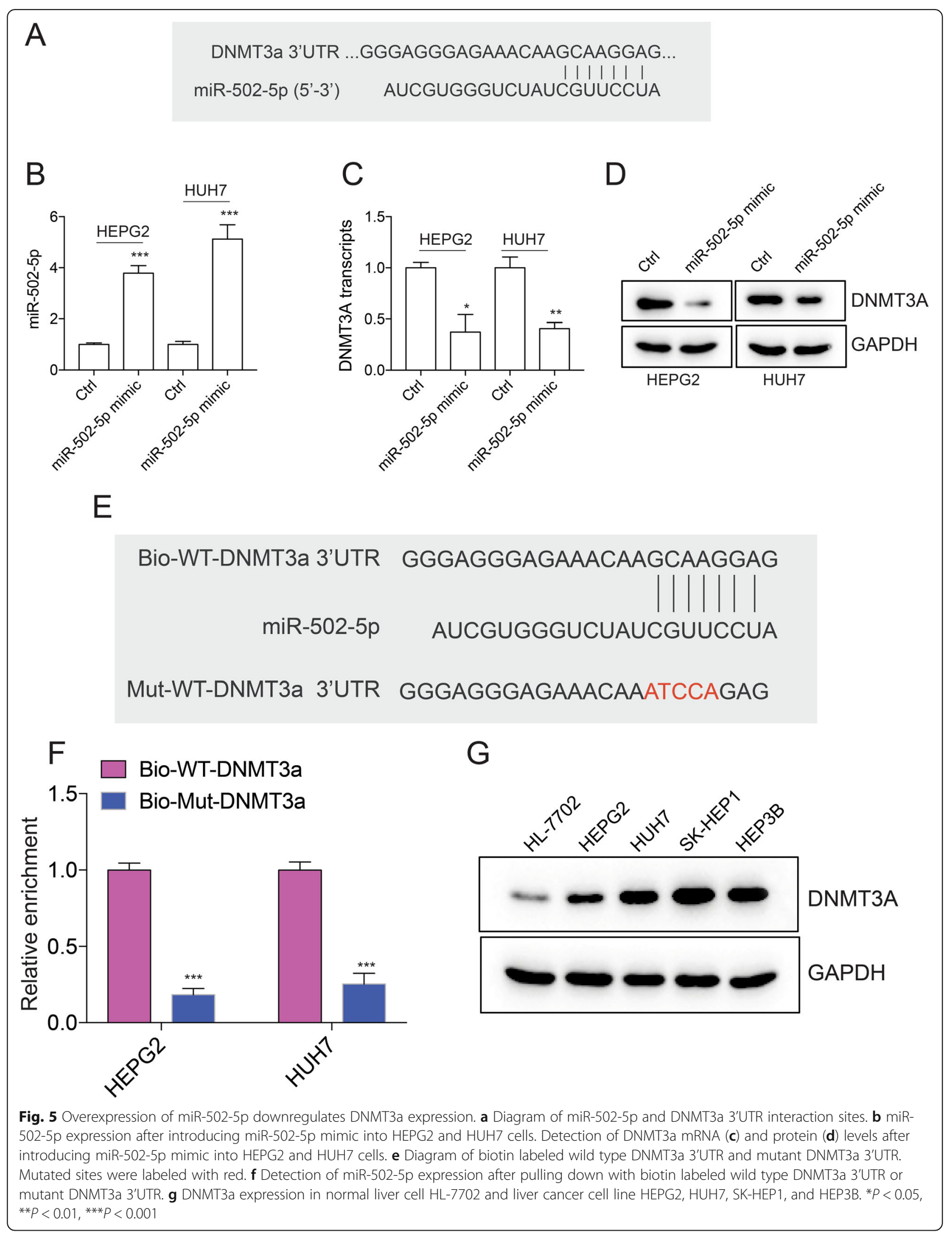




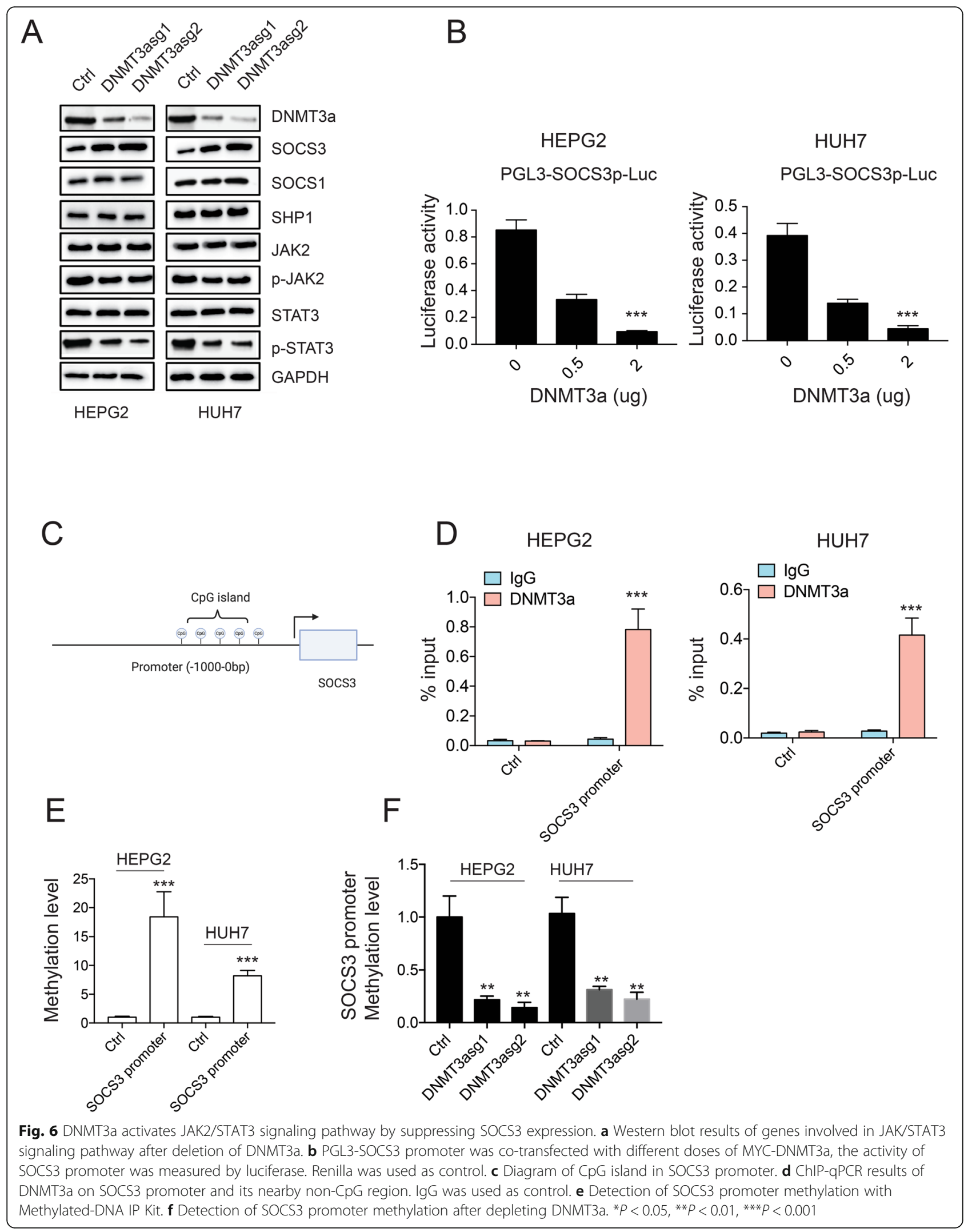




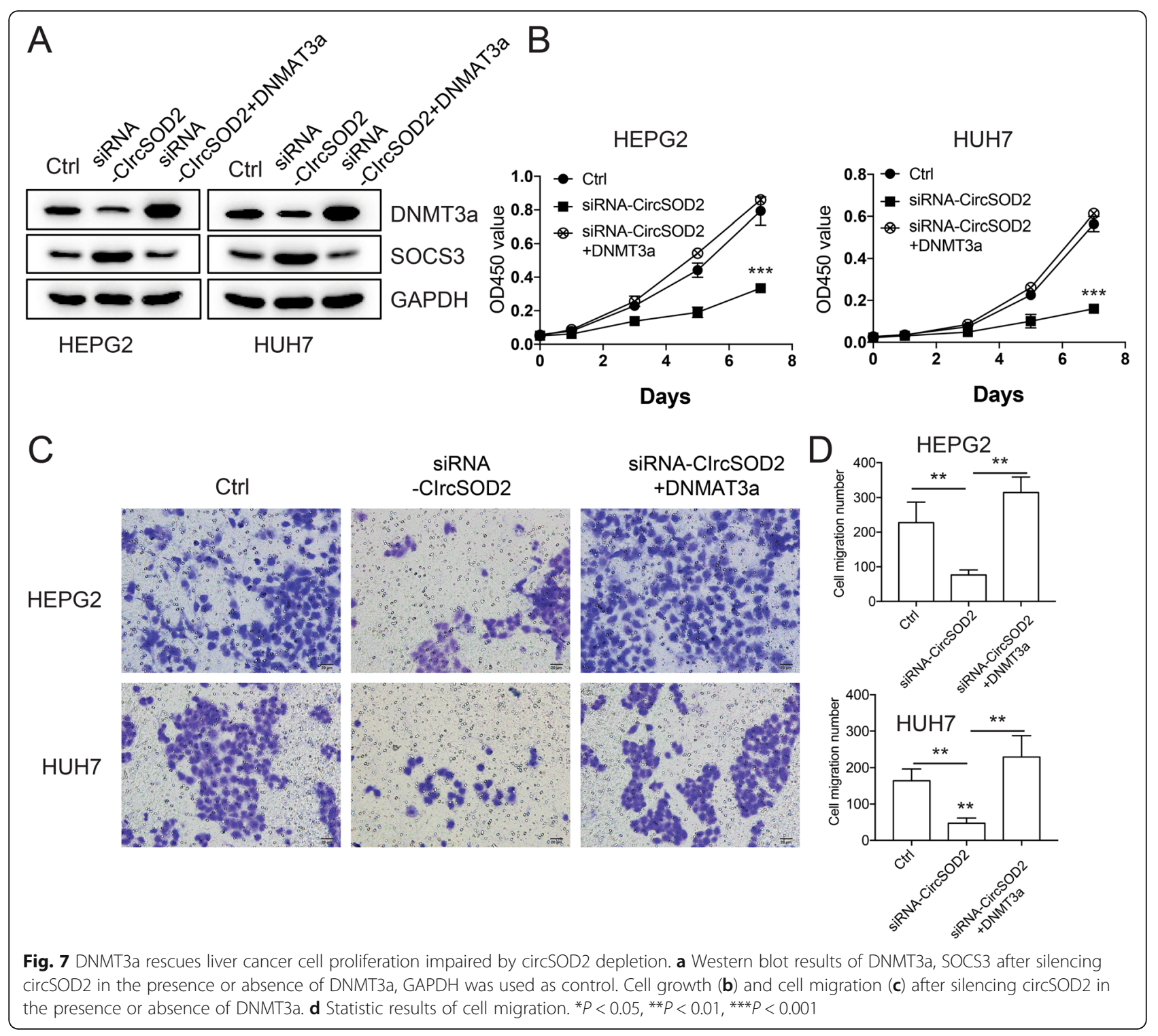

enhanced early cell apoptosis and inhibited cell proliferation [36]. However, upregulation of miR-502 has been reported to accelerate esophageal cancer cell TE1 proliferation by promoting AKT phosphorylation [37]. In this study, we found that, similar to breast cancer and colon cancer, miR-502-5p was also downregulated in HCC. Further studies on circSOD2 and miR-502-5p showed that miR-502-5p binds to circSOD2 exon 3, depletion of circSOD2 significantly upregulated miR-502-5p expression. These results suggest that in HCC, miR-502-5p was suppressed by circSOD2 through direct interaction.

Bioinformatic analysis showed that miR-502-5p binds to DNMT3a 3'UTR. Introducing miR-502-5p into liver cancer cells greatly suppressed DNMT3a expression, suggesting that DNMT3a is a target of miR-502-5p in HCC. The importance of DNMT3a in HCC progression has been extensively studied. Zhao et al., found that depletion of DNMT3a suppressed HCC cell proliferation [38]. Chen et al., reported that miR-30a-3p inhibits the proliferation of liver cancer cells by targeting DNMT3a [39]. Similarly, in this study we also demonstrated that downregulation of DNMT3a by silencing circSOD2 impaired liver cancer cell growth and migration.

DNMT3a mediated DNA hypermethylation suppressed gene expression and has been associated with many cancer developments. DNA hyper-methylation at NF2 and KIBRA promoter mediated by MOC2 and DNMT3a complexes suppresses their gene expression and Hippo signaling pathway activation, which further promotes HCC cancer stemness and tumorigenesis [40]. In Triple Negative Breast Cancer, coupled with MYC, DNMT3a promotes the epithelial to mesenchymal transition and mammosphere formation of TNBC cells by upregulating miR-200b promoter methylation [41]. SUV39H1/DNMT3A-dependent 


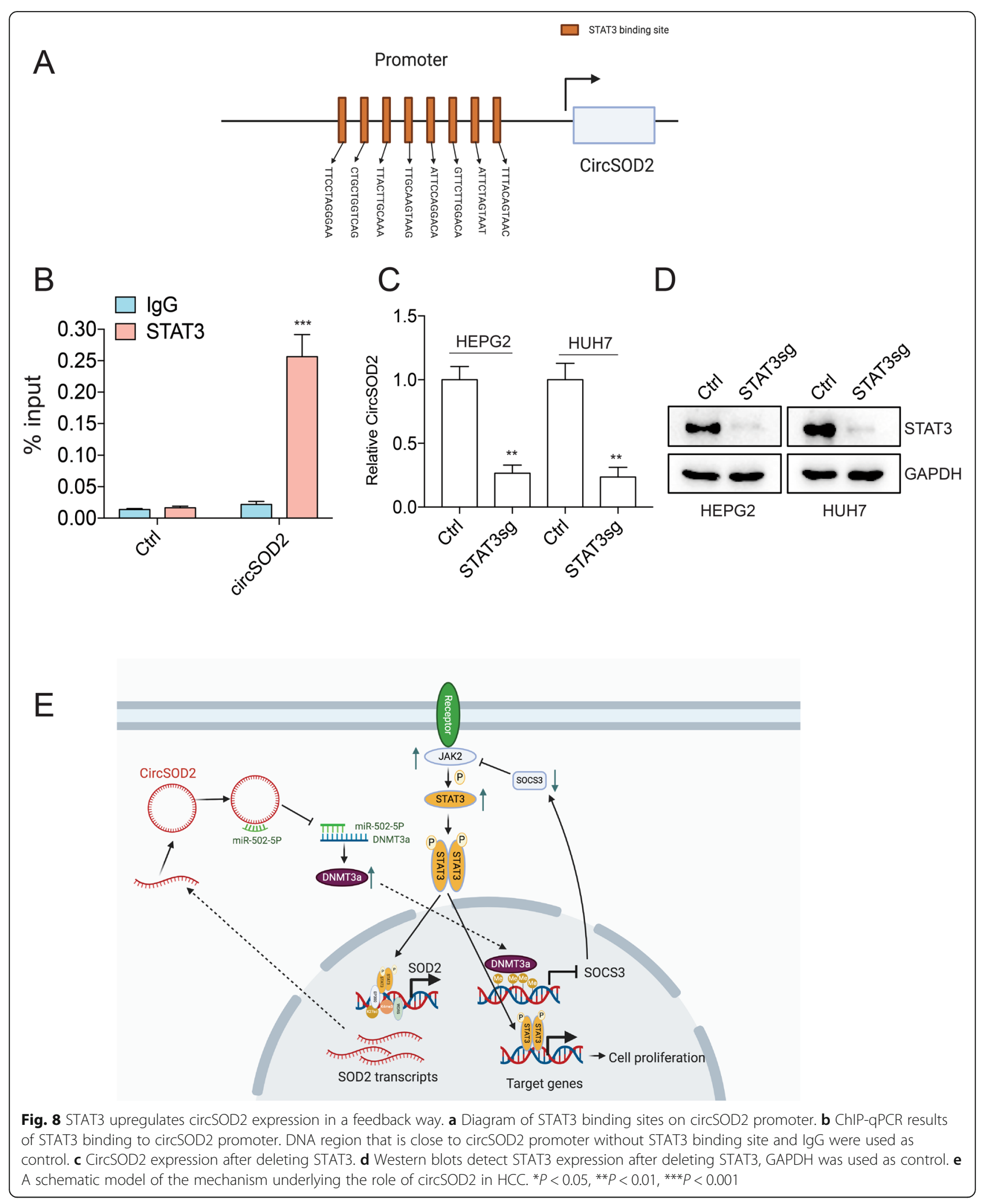


methylation of the RB1 promoter stimulates PIN1 expression and melanoma development [42]. Here, by knocking down DNMT3a, we discovered that SOCS3 is regulated by DNMT3a. Further analysis and experimental validation demonstrated that upregulated DNMT3a in liver cancer cells promoted SOCS3 promoter hyper-methylation and suppressed SOCS3 expression.

Abnormal activation of IL-6/STAT3 signaling pathway has been implicated in the progression of HCC. In HBx induced hepatocellular carcinoma mice model, IL-6/ STAT3, and Wnt/ $\beta$-catenin signaling pathways are constitutively activated [43]. Tumor cell-intrinsic Tim-3 promotes liver cancer via NF- $\mathrm{kB} / \mathrm{IL}-6 / \mathrm{STAT} 3$ axis [34]. Long non-coding RNA DILC regulates liver cancer stem cells via IL-6/STAT3 axis [32]. In this study, we uncovered a new mechanism through which STAT3 is activated, we showed that, SOCS3 suppression mediated by DNMT3a methylation, activated JAK2/STAT3 signaling pathway. Due to the complexity of IL-6/STAT3 signaling pathway, more studies should be performed to clarify the crosstalk among different pathways.

We also evaluated the mechanism through which circSOD2 was activated in HCC. We found that by tethering to circSOD2 promoter, EP300 and WDR5 facilitated circSOD2 promoter H3K27ac and H3K4me3 modification respectively, and increased circSOD2 expression. Moreover, STAT3 binding sites on circSOD2 promoter recruited STAT3 binding to circSOD2 promoter and upregulated circSOD2 expression in a feedback way. Although, for now, we do not have any idea whether STAT3 recruits EP300 or WDR5 into circSOD2 promoter or vice versa, more studies should be performed in the future to clarify this.

\section{Conclusions}

In summary, our clinical data, in vitro cell assay, and tumor formation support the notion that circSOD2 plays a critical role in HCC progression. circSOD2/miR-5025p/DNMT3a/SOCS3-JAK2/STAT3/circSOD2 axis, a novel pathway through which JAK2/STAT3 is activated provides promising therapeutic targets against HCC.

\section{Supplementary Information}

The online version contains supplementary material available at https://doi. org/10.1186/s13046-020-01769-7.

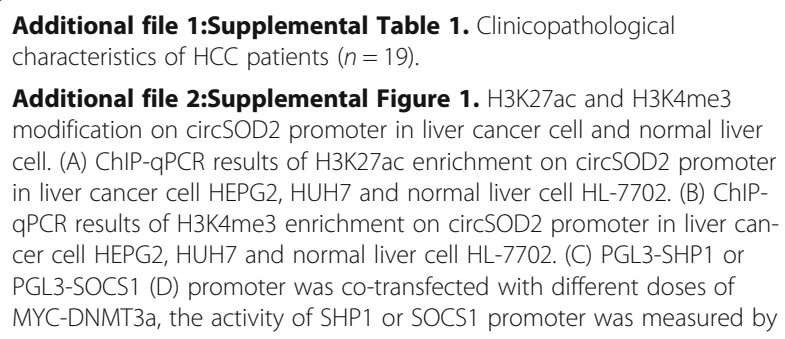

luciferase. Renilla was used as control. (E) The correlation of circSOD2 and SOCS3 in HCC tissues. (F) The correlation of circSOD2 and DNMT3a in HCC tissues. ${ }^{*} P<0.05,{ }^{* *} P<0.01,{ }^{* *} P<0.001$.

\section{Abbreviations}

MiRNA: Micro RNA; LncRNA: Long non-coding RNA; HCC: Hepatocellular carcinoma; ChIP-qPCR: Chromatin Immunoprecipitation quantitative real time PCR; CircRNA: Circular RNA; qRT-PCR: Real-Time Quantitative Reverse Transcription PCR; PI: Propidium iodide; RIP: RNA immunoprecipitation; NC: Nitrocellulose membrane; RIPA: Radioimmunoprecipitation assay; $3^{\prime}$ UTR: 3' untranslated region

\section{Acknowledgements}

We would like to thank Min Xu for her insightful discussion.

\section{Authors' contributions}

ZWZ was responsible for tissue collection, in vitro cell operation and first manuscript draft. JJS performed cell transfection. DKZ and FZW also collected tissue specimen, JFT and LYZ analyzed the data and data validation. JSJ contributed to study design, data interpretation and final manuscript approval. BFT and SJF helped us with the sgRNA design for DNMT3a, LYZ, YG, CMC helped us with the siRNA work on circSOD2, XHH, QYW, YY helped us with the cell growth, and manuscript proof. All authors read and approved the final manuscript.

\section{Funding}

This study was supported by the National Key Research and Development projects intergovernmental cooperation in science and technology of China (2018YFE0126900), and the Provincial and ministerial joint construction of key projects (No. WKJ-ZJ-1932), and the Natural Science Foundation of Zhejiang Province (Nos. LQ20H160056, LQ20H160055), and the Public welfare projects of Zhejiang Province (Nos. LGF19H180010, LGD19H160002 and LGF19H180009), and the Medical and Health Research Project of Zhejiang Province (Nos. 2018KY933 and 2020KY380), and the Key R\&D Program of Lishui City (No. 2019ZDYF17).

\section{Availability of data and materials}

The datasets used and/or analyzed during the current study were available from the corresponding authors on reasonable request.

\section{Ethics approval and consent to participate}

The human cancer tissues used in this study were approved by the institute. Ethical committee of Fifth Affiliated Hospital of Wenzhou Medical University. All patients gave written informed consent.

\section{Consent for publication}

The consent forms were signed by every participant and will be provided upon request.

\section{Competing interests}

Authors declare that there is no conflict interest

Received: 5 August 2020 Accepted: 8 November 2020

Published online: 25 November 2020

\section{References}

1. Global Burden of Disease Cancer, C, et al. Global, Regional, and National Cancer Incidence, Mortality, Years of Life Lost, Years Lived With Disability, and Disability-Adjusted Life-Years for 29 Cancer Groups, 1990 to 2017, A systematic analysis for the global burden of disease study. JAMA Oncol. 2019;5(12):1749-68.

2. El-Serag HB, Rudolph KL. Hepatocellular carcinoma: epidemiology and molecular carcinogenesis. Gastroenterology. 2007;132(7):2557-76.

3. Tang A, et al. Epidemiology of hepatocellular carcinoma: target population for surveillance and diagnosis. Abdom Radiol (NY). 2018;43(1):13-25.

4. Altekruse SF, McGlynn KA, Reichman ME. Hepatocellular carcinoma incidence, mortality, and survival trends in the United States from 1975 to 2005. J Clin Oncol. 2009;27(9):1485-91. 
5. Finn RS, et al. Therapies for advanced stage hepatocellular carcinoma with macrovascular invasion or metastatic disease: a systematic review and metaanalysis. Hepatology. 2018;67(1):422-35.

6. Schmidt-Arras D, Rose-John S. IL-6 pathway in the liver: from physiopathology to therapy. J Hepatol. 2016;64(6):1403-15.

7. Wang $Y$, et al. The long noncoding RNA IncTCF7 promotes self-renewal of human liver cancer stem cells through activation of Wnt signaling. Cell Stem Cell. 2015;16(4):413-25.

8. Khemlina $G$, Ikeda S, Kurzrock R. The biology of hepatocellular carcinoma: implications for genomic and immune therapies. Mol Cancer. 2017;16(1):149.

9. Ferracin $\mathrm{M}$, et al. Circulating non-coding RNA as biomarkers in colorectal Cancer. Adv Exp Med Biol. 2016;937:171-81.

10. Chen B, Huang S. Circular RNA: an emerging non-coding RNA as a regulator and biomarker in cancer. Cancer Lett. 2018;418:41-50.

11. Tang Q, Hann SS. HOTAIR: an oncogenic long non-coding RNA in human Cancer. Cell Physiol Biochem. 2018;47(3):893-913.

12. Romano G, et al. Small non-coding RNA and cancer. Carcinogenesis. 2017; 38(5):485-91.

13. Anastasiadou E, Jacob LS, Slack FJ. Non-coding RNA networks in cancer. Nat Rev Cancer. 2018;18(1):5-18.

14. Zhang $X O$, et al. Diverse alternative back-splicing and alternative splicing landscape of circular RNAs. Genome Res. 2016;26(9):1277-87.

15. Qiu L, et al. Circular RNA profiling identifies circADAMTS13 as a miR-484 sponge which suppresses cell proliferation in hepatocellular carcinoma. Mol Oncol. 2019;13(2):441-55.

16. Xie B, et al. CircRNA has_circ_0078710 acts as the sponge of microRNA-31 involved in hepatocellular carcinoma progression. Gene. 2019;683:253-61.

17. Hsiao KY, Sun HS, Tsai SJ. Circular RNA - new member of noncoding RNA with novel functions. Exp Biol Med (Maywood). 2017;242(11):1136-41.

18. Zhang $M$, et al. A novel protein encoded by the circular form of the SHPRH gene suppresses glioma tumorigenesis. Oncogene. 2018;37(13):1805-14.

19. Wesselhoeft RA, Kowalski PS, Anderson DG. Engineering circular RNA for potent and stable translation in eukaryotic cells. Nat Commun. 2018;9(1): 2629

20. Pamudurti NR, et al. Translation of CircRNAs. Mol Cell. 2017;66(1):9-21 e7.

21. Holdt LM, et al. Circular non-coding RNA ANRIL modulates ribosomal RNA maturation and atherosclerosis in humans. Nat Commun. 2016;7:12429.

22. Yu J, et al. Circular RNA cSMARCA5 inhibits growth and metastasis in hepatocellular carcinoma. J Hepatol. 2018;68(6):1214-27.

23. Hsiao KY, et al. Noncoding effects of circular RNA CCDC66 promote Colon Cancer growth and metastasis. Cancer Res. 2017;77(9):2339-50.

24. Zhao ZJ, Shen J. Circular RNA participates in the carcinogenesis and the malignant behavior of cancer. RNA Biol. 2017;14(5):514-21.

25. Vo JN, et al. The landscape of circular RNA in Cancer. Cell. 2019:176(4):869 81 e13.

26. Luo Z, Mao X, Cui W. Circular RNA expression and circPTPRM promotes proliferation and migration in hepatocellular carcinoma. Med Oncol. 2019; 36(10):86.

27. Igolkina AA, et al. H3K4me3, H3K9ac, H3K27ac, H3K27me3 and H3K9me3 Histone Tags Suggest Distinct Regulatory Evolution of Open and Condensed Chromatin Landmarks. Cells. 2019;8(9):1034

28. Zubritskiy A, Medvedeva YA. DNA sequence features in the establishing of H3K27ac. F1000Res. 2018;7:165. https://doi.org/10.12688/f1000research.13441.2.

29. Panda AC. Circular RNAs act as miRNA sponges. Adv Exp Med Biol. 2018; 1087:67-79.

30. Piwecka $\mathrm{M}$, et al. Loss of a mammalian circular RNA locus causes miRNA deregulation and affects brain function. Science. 2017;357(6357).

31. Ha M, Kim VN. Regulation of microRNA biogenesis. Nat Rev Mol Cell Biol. 2014;15(8):509-24.

32. Wang $X$, et al. Long non-coding RNA DILC regulates liver cancer stem cells via IL-6/STAT3 axis. J Hepatol. 2016;64(6):1283-94.

33. Yin Z, et al. IL-6/STAT3 pathway intermediates M1/M2 macrophage polarization during the development of hepatocellular carcinoma. J Cell Biochem. 2018;119(11):9419-32.

34. Zhang $\mathrm{H}$, et al. Tumor cell-intrinsic Tim-3 promotes liver cancer via NFkappaB/IL-6/STAT3 axis. Oncogene. 2018;37(18):2456-68.

35. Zhai $\mathrm{H}$, et al. Inhibition of autophagy and tumor growth in colon cancer by miR-502. Oncogene. 2013;32(12):1570-9.

36. Sun $L L$, et al. Suppressive role of miR-502-5p in breast cancer via downregulation of TRAF2. Oncol Rep. 2014;31(5):2085-92.
37. Xu J, Pan X, Hu Z. MiR-502 mediates esophageal cancer cell TE proliferation by promoting AKT phosphorylation. Biochem Biophys Res Commun. 2018;501(1):119-23.

38. Zhao Z, et al. Depletion of DNMT3A suppressed cell proliferation and restored PTEN in hepatocellular carcinoma cell. J Biomed Biotechnol. 2010; 2010:737535.

39. Chen $\mathrm{Q}$, et al. miR-30a-3p inhibits the proliferation of liver cancer cells by targeting DNMT3a through the PI3K/AKT signaling pathway. Oncol Lett. 2020;19(1):606-14

40. Wang T, et al. Epigenetic restriction of hippo signaling by MORC2 underlies stemness of hepatocellular carcinoma cells. Cell Death Differ. 2018;25(12): 2086-100.

41. Pang $Y$, et al. MYC and DNMT3A-mediated DNA methylation represses microRNA-200b in triple negative breast cancer. J Cell Mol Med. 2018;22(12): 6262-74.

42. Kim G, et al. SUV39H1/DNMT3A-dependent methylation of the RB1 promoter stimulates PIN1 expression and melanoma development. FASEB J. 2018:32(10):5647-60.

43. Wang $C$, et al. Hepatitis B virus $X(H B x)$ induces tumorigenicity of hepatic progenitor cells in 3,5-diethoxycarbonyl-1,4-dihydrocollidine-treated HBX transgenic mice. Hepatology. 2012;55(1):108-20.

\section{Publisher's Note}

Springer Nature remains neutral with regard to jurisdictional claims in published maps and institutional affiliations.
Ready to submit your research? Choose BMC and benefit from:

- fast, convenient online submission

- thorough peer review by experienced researchers in your field

- rapid publication on acceptance

- support for research data, including large and complex data types

- gold Open Access which fosters wider collaboration and increased citations

- maximum visibility for your research: over $100 \mathrm{M}$ website views per year

At BMC, research is always in progress.

Learn more biomedcentral.com/submissions 